\title{
Detecção e caracterização automatizada de fraturas geológicas em perfil acústico
}

*Luciana Olivia Dias (CBPF), Clécio R. De Bom (CBPF/CEFET), Márcio Albuquerque (CBPF), Marcelo Albuquerque (CBPF), Elisângela Faria (CBPF) e Maury Correia (CENPES/Petrobrás)

Copyright 2016, SBGf - Sociedade Brasileira de Geofísica

Este texto foi preparado para a apresentação no VII Simpósio Brasileiro de Geofísica. Ouro Preto, 25 a 27 de outubro de 2016. Seu conteúdo foi revisado pelo Comitê T́́ Tecnico do VII SimBGr, mas não necessariamente representa a opinia pe da SBGt ou de seus associados. E proibida a reprodução total ou parcial deste material para propósitos comerciais sem prévia autorização da SBGf.

\section{Resumo}

Detecting Fractures is an essential task in borehole image analysis. The knowledge of the location, direction and inclination of fractures of the pit wall allows the development of exploitation strategies that can enhance well production. The studies of the production history of fractured reservoirs show that the fractures play an important role in the productivity of formations with permeability. Typically an specialist takes considerable working time to determine them by visual inspection or to confirm some fractures suggested by commercial software. In this contribution we present an algorithm to detect fractures in an automated fashion. We compare the method with other proposed implementations to detect fractures.

\section{Introdução}

A determinação da viabilidade de extração de recursos naturais de interesse depende do conhecimento e da análise de ocorrências geológicas que estão diretamente ligadas a produção do reservatório (Hornby e Luthi, 1992). Na maioria dos casos as fraturas tectônicas de interesse para a produção são aquelas que apresentam uma abertura ou um preenchimento com material que possuem propriedades físicas diferentes da rocha matriz (Ellis e Singer, 2008). Em uma formação rochosa natural fraturas também podem apontar a direção de escoamento e potencial armazenamento. Para fins de avaliação de formação, uma estrutura de regime rúptil é auxiliar na definição de características de porosidade e permeabilidade do reservatório (Ellis e Singer, 2008).

Com a finalidade de avaliar se o solo de uma região é propicia a reservatório, são utilizadas ferramentas que perfuram e perfilam a parede de um poço (Luthi, 2001). Em especial perfis de imagens acústicas UBI (Ultrasonic Borehole Imager), nas quais são possíveis avaliar diferentes texturas e estruturas, tais como fraturas que compõe a rocha adjacente possibilitando uma avaliação (Ellis e Singer, 2008). Interpretando de forma geométrica, a abertura de uma fratura ao ser interceptada por um poço, pode ser vista como dois planos interceptando um cilindro (Luthl, 2001 e Zoback, 1985). Logo a imagem obtida, confere a figura planar dessa interpretação geométrica que considera um padrão à fratura semelhante a uma senóide (Luthl, 2001; Gaillot, 2007). Porém, essa interpretação nem sempre é real e não torna trivial a classificação dessa ocorrência entre todo um complexo sistema de formações existente no solo.

Neste trabalho propomos um algoritmo automatizado para detectar nas imagens acústicas, regiões de possíveis fraturas. O algoritmo proposto é inspirado em Xavier e Andrade, 2015. Fazemos também uma comparação com outras propostas de detecção de fraturas encontradas na literatura (Cornet, Jan; 2013).

\section{Metodologia/ Problema Investigado}

O algoritmo de detecção de fraturas é composto por 8 etapas, figura 1 , a seguir comentamos cada uma. Para realizar detecção de fraturas em um grande volume de dados é necessário realizar pré-processamento para reduzir o conjunto de dados a serem analisados. Este processo é fundamental para reduzir o custo computacional de qualquer método que procure encontrar e fazer medidas em candidatos a fraturas. Esta etapa consiste em realçar o contraste na imagem inteira. A seguir, são identificados trechos que apresentam curva com níveis mais altos dessa característica, visto na figura 2. Na etapa seguinte nas regiões de maior contraste são realizadas as segmentações utilizando método de clustering K-means (Chai, Hum Y.; 2011). Ao separar as estruturas do fundo a imagem é binarizada e um filtro de conectividade é utilizado para que os objetos então possam ser rotulados. A seguir, ajustamos os objetos que restaram e satisfaçam todas as etapas a uma curva de polinômio de $4^{\circ}$ grau (Xavier e Andrade, 2015). As curvas ajustadas, nesta etapa com um coeficiente de correlação maior que um limiar determinado pelo usuário são consideradas fraturas. Neste trabalho utilizamos como limiar 0.9. A saída do algoritmo é uma lista de fraturas e a localização em profundidade. Também com esses dados é possível obter a atributos de abertura, inclinação e a orientação dessas ocorrências. A metodologia apresentada foi aplicada em imagem real, de dados de amplitude pertencentes a trecho de poço (Cornet, Jan; 2013), como visto no exemplo da figura 3. Mas, como a quantidade de dados para validação do modelo é muito limitada, desenvolvemos imagens sintéticas originadas do dado real modificado, utilizando uma técnica conhecida como image quilting (Efros e Freeman, 2001) simulando a rocha matriz de fundo. Essa técnica gera vários tipos de combinações de texturas a partir de pequenas áreas da imagem original. As fraturas sintéticas inseridas sobre esse fundo foram desenvolvidas a partir dos valores de amplitudes de fratura real recombinadas. Dessa forma, comparamos o modelo apresentado com outras técnicas de detecção de fraturas geológicas já referenciadas na literatura: Transformada de Hough (Ballard, Dana; 1981), Transformada de Radon (Starck, Jean-Luc; 2002) e Correlation (Sutton, M.;1986). 


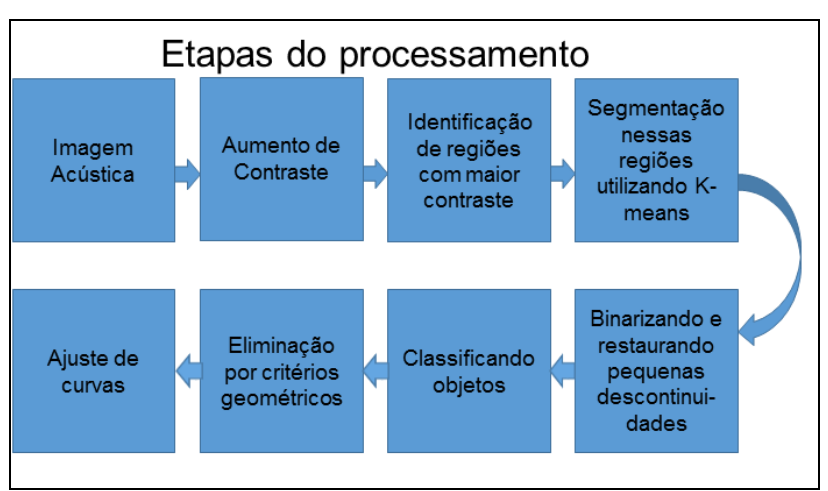

Figura 1 - Esquema representa os processos executados pelo código de detecção de fraturas.

\section{Resultados}

Foram geradas aleatoriamente 50 imagens com fraturas sintéticas de senos ou cossenos com valores de amplitudes variando de 0.05 a $0.3 \mathrm{com}$ fases variando de -pi/4 a pi/4 e frequência angular no intervalo de a 0.7 a 1.3. Exemplo dessas fraturas podem ser vistas na figura 4. $\mathrm{Na}$ segunda etapa representamos ambientes geológicos típicos dos reservatórios. Utilizando o mesmo método aplicado na imagem real em imagens sintéticas podemos obter número de fraturas encontradas. As imagens de amplitudes acústicas modeladas possuem as texturas similares de uma imagem real. Também realizamos testes com uma ou mais fraturas próximas para avaliar como o algoritmo se comporta em ambientes com múltiplas fraturas. A distribuição de nível de intensidade dos pixels da fratura também reproduz a distribuição da fratura real. O mesmo efeito de contraste nas fraturas reais é observado nas simuladas. Esse efeito também é observado em ocorrências como breakouts e marcas de ferramenta de perfil devido aos baixos valores de amplitude comparados com as da rocha matriz, i.e, fundo da imagem. Mas, essas ocorrências possuem geometria diferenciada das fraturas e são excluídas durante o processo. Após obter os percentuais de acertos de cada técnica, podemos constatar que a metodologia proposta obtém um percentual acima de $70 \%$ nas imagens sintéticas. Com a Transformada de Hough $0 \%$, Transformada de Randon $0 \%$ e com o Método de Correlação $0 \%$ de acertos nas imagens sintéticas.

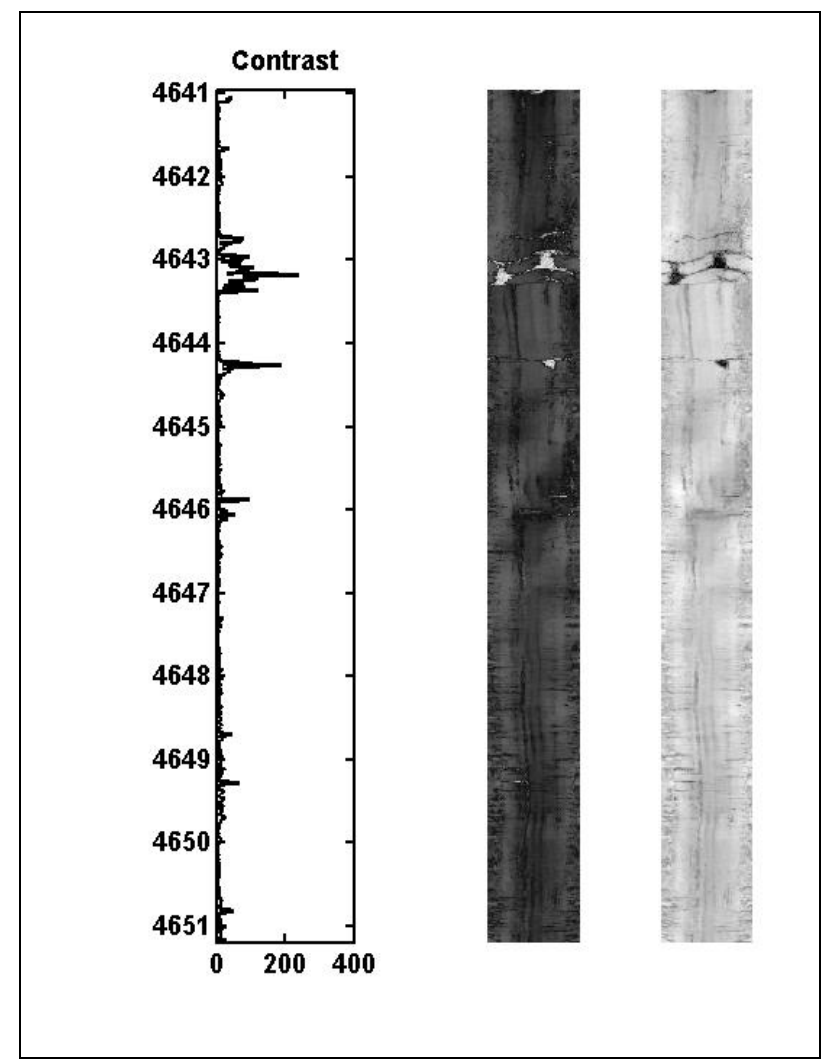

Figura 2 - Indicador de regiões de maior contraste da imagem resultado do pré-processamento (B). Entre o gráfico e a imagem resultado está a imagem em estado original em tons de cinza $(A)$.

\section{Discussão e Conclusões}

Neste trabalho apresentamos um algoritmo para detecção de fraturas de maneira automatizada. Aplicamos a técnica em imagens simuladas de parâmetros diversos. Foi possível comparar então com outras três técnicas disponíveis na literatura Transformada de Hough, Transformada de Randon e o Método de Correlação. Essa simulação das imagens acústicas de amplitude apresentada é capaz de representar uma multiplicidade de cenários geológicos representativos dos reservatórios fraturados. A metodologia adotada obteve sucesso qualitativo na identificação no exemplo da imagem real, visto na figura 3 , como nas imagens sintéticas, conforme observado na figura 4 , verificando que o ajuste polinomial das bordas da fratura não apresenta a limitação da representação geométrica de uma fratura por uma senóide, mas se ajusta a uma geometria de poço ovalizado. O estudo foi realizado tanto nas imagens sintéticas, como no processamento, para as imagens reais, confrontando os resultados obtidos com a interpretação feita em outros métodos. Estes métodos são falhos com imagens de baixo contraste. Mas, esse estudo não se limita a essas condições, um número maior de dados de poços reais para validação é necessário para caracterizar 
adequadamente a população de falsos positivos e atestar a robustez da técnica.

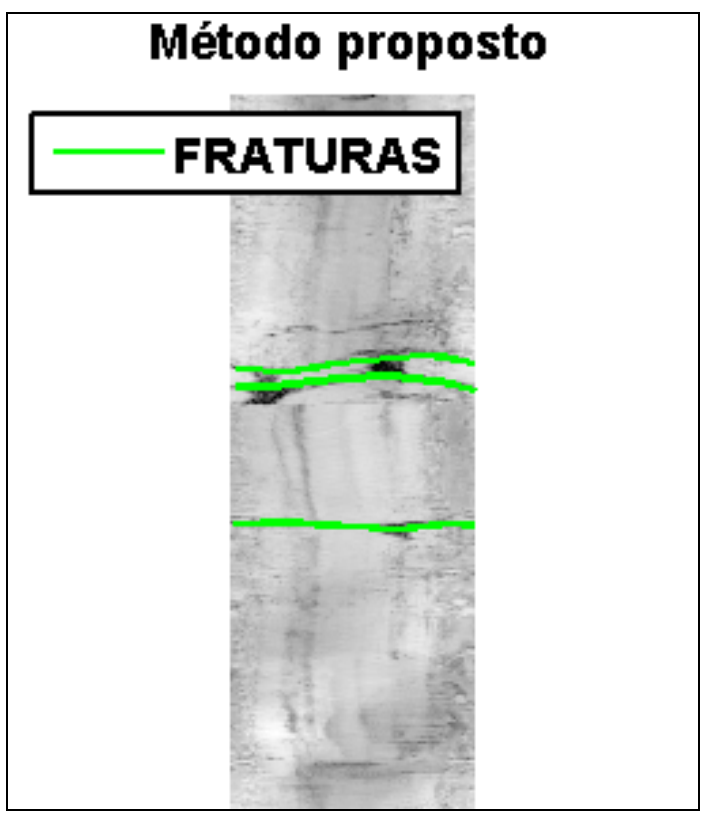

Figura 3 - Nessa figura podemos visualizar o resultado final nesse trecho de imagem real. Observamos que as curvas foram devidamente ajustadas.

\section{Agradecimentos}

Este trabalho foi possível através da cooperação entre o CBPF e o CENPES e recebeu apoio da rede CARMOD, uma rede temática para fomento de pesquisa na área de carbonatos.

\section{Referências}

Al-Sit, Waleed. Automatic feature detection and interpretation in borehole data. Diss. University of Liverpool, 2015.

Cornet, Jan. "Fracture detection and analysis from image log raw data." (2013).

EFROS, Alexei A.; FREEMAN, William T. Image quilting for texture synthesis and transfer. In: Proceedings of the 28th annual conference on Computer graphics and interactive techniques. ACM, 2001. p. 341-346.

ELLIS, D.V; SINGER, J.M. Well logging for earth scientists. New York: Elsevier, 2008.

GAILLOT, P.; BREWER, T. PEZARD, P. YEH, E C. Borehole Imaging Tools - Principles and applications.
Technical Developments. Scientific Drilling. № 05. Set, 2007.

HORNBY, Brian E. LUTHI, Stefan M. Na integrated interpretation of fracture apertures computed from electral borehole scans and reflected Stonoley waves. Geological Society, London, Special Publication, v. 65, p 185-198. 1992.

LUTHI, S. M. Geological well logs: their use in reservoir modeling. Berlin, SpringerVerlag. 373p. 2001.

ROTHKOPF, B. WADLEIGH, E. Re-tooling Improves Field Efficiency. The American Oil and Gas Reporter, $p$ 91-95. Set 1994.

ZOBACK, M.D., MOOS, D., MASTIN, L., ANDERSON, R.N. Wellbore breakouts and in situ stress. Journal of Geophysical Research 90 (B7), 5523-5530. 1985.

Ruela, A., and C. Guerra. Detecção de fraturas em imagem de perfis geofísicos de poço com morfologia matemática. III Simpósio Brasileiro de Ciências Geodésicas e Tecnologias da Geoinformação, Recife, Brasil (2010): 21-30.

Chai, Hum Y., et al. "Gray-level co-occurrence matrix bone fracture detection." American Journal of Applied Sciences 8.1 (2011): 26.

Ballard, Dana H. "Generalizing the Hough transform to detect arbitrary shapes." Pattern recognition 13.2 (1981): 111-122.

Starck, Jean-Luc, Emmanuel J. Candès, and David L. Donoho. "The curvelet transform for image denoising." Image Processing, IEEE Transactions on11.6 (2002): 670-684.

Sutton, M. A., et al. "Application of an optimized digital correlation method to planar analysis." Image and Vision Computing 4.3 (1986): 143-150. 


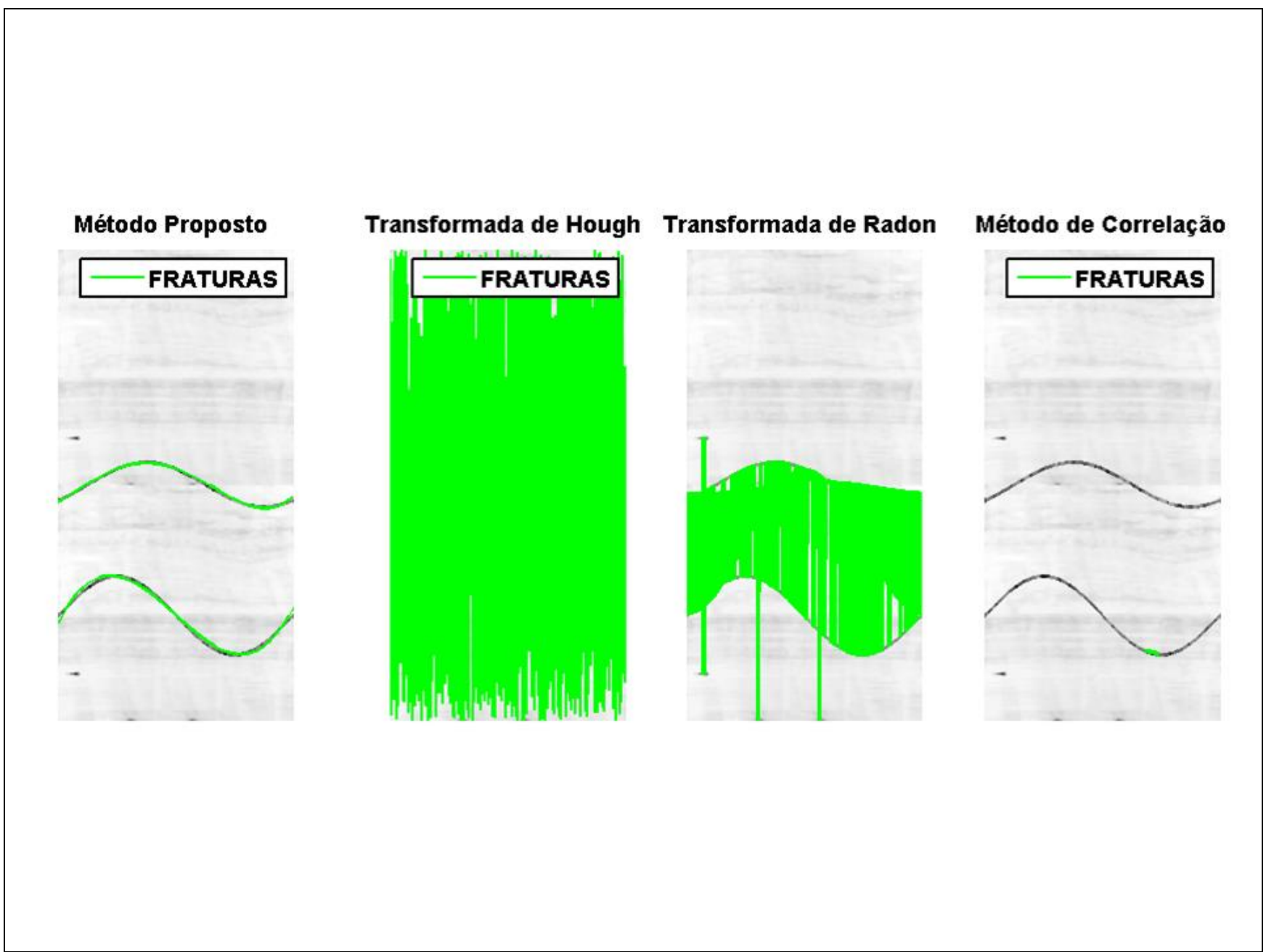

Figure 4 - Exemplo de resultado obtido por cada método. A linha em verde delimita as fraturas encontrada por cada método. O proposto conseguiu detectar com sucesso as fraturas na imagem sintética, obtendo um coeficiente de correlação entres os dados e a curva superior a 0.9 , bem próximo a 1 o melhor valor de ajuste. Os demais não foram capazes de detectar as fraturas devido ao baixo contraste com o fundo. 